\title{
Why hot horizontal branch stars can appear redder than red giants ${ }^{\star}$
}

\author{
Y. Momany ${ }^{1}$, S. Cassisi ${ }^{2}$, G. Piotto ${ }^{1}$, L. R. Bedin ${ }^{1}$, S. Ortolani ${ }^{1}$, F. Castelli ${ }^{3,4}$, and A. Recio-Blanco ${ }^{1}$ \\ ${ }^{1}$ Dipartimento di Astronomia, Università di Padova, Vicolo dell'Osservatorio 2, 35122 Padova, Italy \\ e-mail: piotto; bedin; ortolani; recio@pd.astro.it \\ 2 INAF - Osservatorio Astronomico di Collurania, via M. Maggini, 64100 Teramo, Italy \\ e-mail: cassisi@te. astro.it \\ 3 Istituto di Astrofisica Spaziale e Fisica Cosmica, CNR, via del Fosso del Cavaliere, 00133 Roma, Italy \\ ${ }^{4}$ Osservatorio Astronomico di Trieste, via Tiepolo 11, 34131 Trieste, Italy \\ e-mail: castelli@ts.astro.it
}

Received 11 March 2003 / Accepted 16 May 2003

\begin{abstract}
In this paper we report on a curious feature in the $V,(U-B)$ color-magnitude diagrams of globular clusters. In our database, we find that a considerable fraction of blue horizontal branch stars, hotter than the instability strip and cooler than the Grundahl et al. (1999) jump (i.e., $\left.6000 \lesssim T_{\text {eff }}(\mathrm{K}) \lessgtr 10000\right)$, have $(U-B)$ colors redder than their red giant progenitors. This red incursion is not expected on theoretical grounds, as horizontal branch stars (whose convective regions are less extended than in red giant structures) should not "appear" cooler than a red giant. Analyzing data from different telescopes we show that: 1) the horizontal branch red incursion is strongly dependent on the shape of the adopted $U$ filter and to a lesser extent, on the $B$ filter; 2) the photometry done with $U$ filters that do not encompass the Balmer jump shows the blue horizontal branch red incursion; 3) the occurrence of this feature is also due to the peculiar dependence of the $U$ and $B$ magnitudes on star effective temperature, gravity, and metallicity; 4) theoretical tracks can reproduce the observed horizontal branch morphology, provided that the appropriate (i.e. exactly responding to the filters effectively used in the observations) transmission curve efficiencies are used for deriving color- $T_{\text {eff }}$ transformations; 5) the red incursion extent depends on metallicity.
\end{abstract}

Key words. stars: imaging - stars: evolution - stars: Hertzsprung-Russell diagram (HR) and C-M diagrams stars: horizontal-branch - ultraviolet: stars

\section{Introduction}

Color-magnitude diagrams (CMD) of resolved stellar populations are the fundamental tool for testing predictions of stellar evolutionary theory and the capability of stellar models to finely reproduce the observations. Photometric studies are no longer limited to the classical BVI bands, as high quality near-infrared and ultraviolet CMDs are becoming more and more available. Ultraviolet (UV) bands certainly cover the ideal wavelength region for the study of hot stellar populations. Indeed, UV observations help to remove the degeneracy between color and temperature for hot blue horizontal branch (HB) stars, allowing a more reliable measurement of atmospheric parameters (in particular temperature estimates).

Recently, the blue horizontal branch (BHB) population has been the subject of specific UV studies, and some interesting features were revealed only in ultraviolet CMDs: jumps

Send offprint requests to: Y. Momany, e-mail: momany@pd. astro. it

* Based on observations with the ESO/MPI $2.2 \mathrm{~m}$ telescope, located at La Silla Observatory (Chile) and on observations with the NASA/ESA Hubble Space Telescope.
(Grundahl et al. 1999, G99; Momany et al. 2002), and peculiar horizontal branch extensions, like the blue-hook (D'Cruz et al. 2000, Brown et al. 2001). The ultimate goal of these studies is a better understanding of the physical mechanisms governing core-helium burning stars, and driving the horizontal branch morphology. To this end, our group has undertaken a long-term project to obtain wide-field multi-wavelength data of BHB globular clusters (GCs). Mainly, we used the WFPC2 on board the HST to map the central core of our target clusters (Piotto et al. 2002), and the wide field imager (WFI) at the ESO/MPI $2.2 \mathrm{~m}$ for the coverage of the outer regions. Unfortunately, observations carried out through non-standard photometric systems sometimes generate puzzling and apparently un-physical properties in the observed CMDs.

In this paper we report on a curious feature in the observed HB morphology in the UV color-magnitude diagrams, and an explanation for it. In some of the $V,(U-B)$ diagrams of our database, we find that the blue HB stars hotter than the instability strip and cooler than the G99 $u$-jump $(-0.1 \lesssim B-V \lesssim 0.2)$ have $U-B$ colors that place them on the right hand side of the red giant branch (RGB). 
According to stellar evolutionary theory blue HB stars should not "appear" cooler than their RGB progenitor. Indeed, HB stars have hotter effective temperatures than RGB stars with the same metallicity. In a color-magnitude diagram, we do not expect any red incursion of BHB stars to the cooler side of the RGB (which almost corresponds to the Hayashi track): for stars in hydrostatic equilibrium and undergoing convection, the Hayashi line separates a permitted region (on its left) from a forbidden one (on its right). Despite this theoretical constraint, we will show that the unexpected finding of the BHB red incursion is present in at least $8 V,(U-B)$ CMDs of BHB clusters we have observed. We will also show that this peculiarity is not present in all the CMDs from broad band photometry of BHB clusters, as the effect depends on the metallicity.

As discussed in Sect. 3, it turns out that the apparent anomaly rises when "non-standard" $U$ filters are used. We decided to publish these color-magnitude diagrams, and discuss the origin of the apparently un-physical HB morphology, in order to call attention to the importance of using appropriate filter sets when acquiring photometric data. Alternatively, and for a proper interpretation of the empirical data, this paper shows the importance of using exactly the photometric passbands of the observational system when transforming the theoretical tracks to the observational plane.

\section{Observations and data reductions}

We present data collected with the Hubble Space Telescope (HST), and the ESO/MPI $2.2 \mathrm{~m}$ telescope. $U B V$ data of NGC 7089 and NGC 5139 were taken on June 2002 with the Wide-Field-Imager (WFI) at the ESO/MPI $2.2 \mathrm{~m}$, employing the "new" $U$ filter (Germany 2002). A second $U B V$ data set of NGC 7078 and NGC 7099 images was obtained on July 2000, employing the "old" $U$ filter at the same telescope.

Basic reduction of the CCD mosaic was performed using the IRAF package MSCRED (Valdes 1998), while stellar photometry was done using DAOPHOT/ALLFRAME (Stetson 1994). Calibration to the standard $U B V$ system was obtained using a set of standard stars from Landolt (1992). HST data of NGC 7099, NGC 7078, and NGC 6205 were taken in the F336W, F439W, and F555W bands. For these clusters we present only instrumental diagrams. Besides the HST and $2.2 \mathrm{~m}$ data, we present $U B V$ published data of NGC 1904 by Kravtsov et al. (1997), and NGC 1841 by Alcaino et al. (1996), both collected at the ESO/NTT telescope. Lastly, we present composite $U B V$ catalogs of NGC 4833 and NGC 4590. The $B V$ photometry of these clusters were taken from Melbourne et al. (2000) and Walker (1994), while the $U$ photometry were obtained employing the WFI "new" $U$ filter at the $2.2 \mathrm{~m}$ telescope.

\section{The blue horizontal branch red incursion}

Figure 1 shows a zoom around the HB in the $V,(B-V)$ and $V$, $(U-B)$ diagrams of the $8 \mathrm{GCs}$, obtained with the 4 different telescopes (HST, NTT, CTIO and $2.2 \mathrm{~m}$ ESO telescope). The clusters span a range in metallicity of $\sim 0.7$ dex, from the metal-poor regime to intermediate metallicity. For all clusters in the sample, the presence of the HB elbow and its incursion through the RGB is quite evident. Due to the larger sample of HB stars, it is really impressive in NGC 7089, NGC 7078, and NGC $5139^{1}$. Note how the blue HB of NGC 7099 lies almost entirely on the red side of the RGB in the $V,(U-B)$ diagram.

Figure 2 shows some of the conflicting results we faced in the course of our investigation. The $V,(U-B)$ diagrams of NGC 7099, from data collected at two different telescopes, show completely different HB morphologies. In the case of the diagram obtained with HST, the BHB incursion is clearly visible, while in the other diagram (based on data collected with the $2.2 \mathrm{~m}$ telescope) the BHB does not cross the cluster RGB at all. These differences are not to be attributed to the limit of the UV region reachable from the ground (at variance with the HST space observations), as Fig. 1 shows two CMDs (for NGC 7089 and NGC 5139), from data obtained at the ESO/MPI $2.2 \mathrm{~m}$ telescope, clearly disclosing the HB red incursion. The effect cannot be attributed to red leakage in the employed $U$ filters (the WFPC2 F336W and WFI new- $U$ filters are known to be affected by red-leaks). Red leakage affects mainly RGB stars, causing them to have smaller than expected $U$ magnitudes and therefore bluer $U-B$ colors. Indeed, considering the F336W filter (the most affected by red leakage among our filters), we calculate that for an RGB star at the level of the $\mathrm{HB}$, with $[\mathrm{Fe} / \mathrm{H}]=-1.8$, the red leakage in the $\mathrm{F} 336 \mathrm{~W}$ band causes a blue-ward shift of only 0.012 mag while the effect we discuss in this paper is of the order of few tenths of a magnitude.

The apparent contradictory results shown in Fig. 2 lead us to search for differences among the filters adopted to acquire the different data sets, in particular at the ESO/MPI $2.2 \mathrm{~m}$ telescope. We found that the $2.2 \mathrm{~m}$ data showing the BHB incursion were obtained using the two "new" $U$ and $B$ filters (Germany 2002), while data not showing the incursion were obtained with the "old" $U$ and $B$ filters.

Prompted by this result, we analyzed the normalized transmission curves of a variety of $U$ and $B$ filters (Fig. 3). We found that the "new" $U$ filter at the $2.2 \mathrm{~m}$, as well as the HST F336W and the Strömgren $u$ filters, have a transmission curve with a peak around $\lambda \simeq 3400 \AA$. On the other hand, the "old" $U$ filter at the $2.2 \mathrm{~m}$, and the $U$-Buser band (Buser 1978) of the synthetic colors (Castelli 1999) that were originally used for the transformations from the theoretical to the observational plane have a transmission curve peaking around $\lambda \simeq 3650 \AA$. It is worth mentioning that the latter group of filters have transmission curves that are closer to the standard $U$ Johnson (although this does not necessarily mean that the observational band, which is a convolution of the filter, CCD, telescope optics, and atmosphere transmission curves, is exactly the standard $U$ Johnson (1955) band). Interestingly enough, the clusters observed with the latter group of filters do not show the BHB incursion, whereas clusters observed through filters whose transmission peak at $\lambda \simeq 3400 \AA$ do. Also the $B$ filter can be important. For example, clusters observed with the new $2.2 \mathrm{~m}$ $U$ filter and the old $2.2 \mathrm{~m} B$ filter (see Fig. 3) do not show the

${ }^{1} I,(U-B)$ diagrams of these clusters show even a greater extent of the red incursion. 

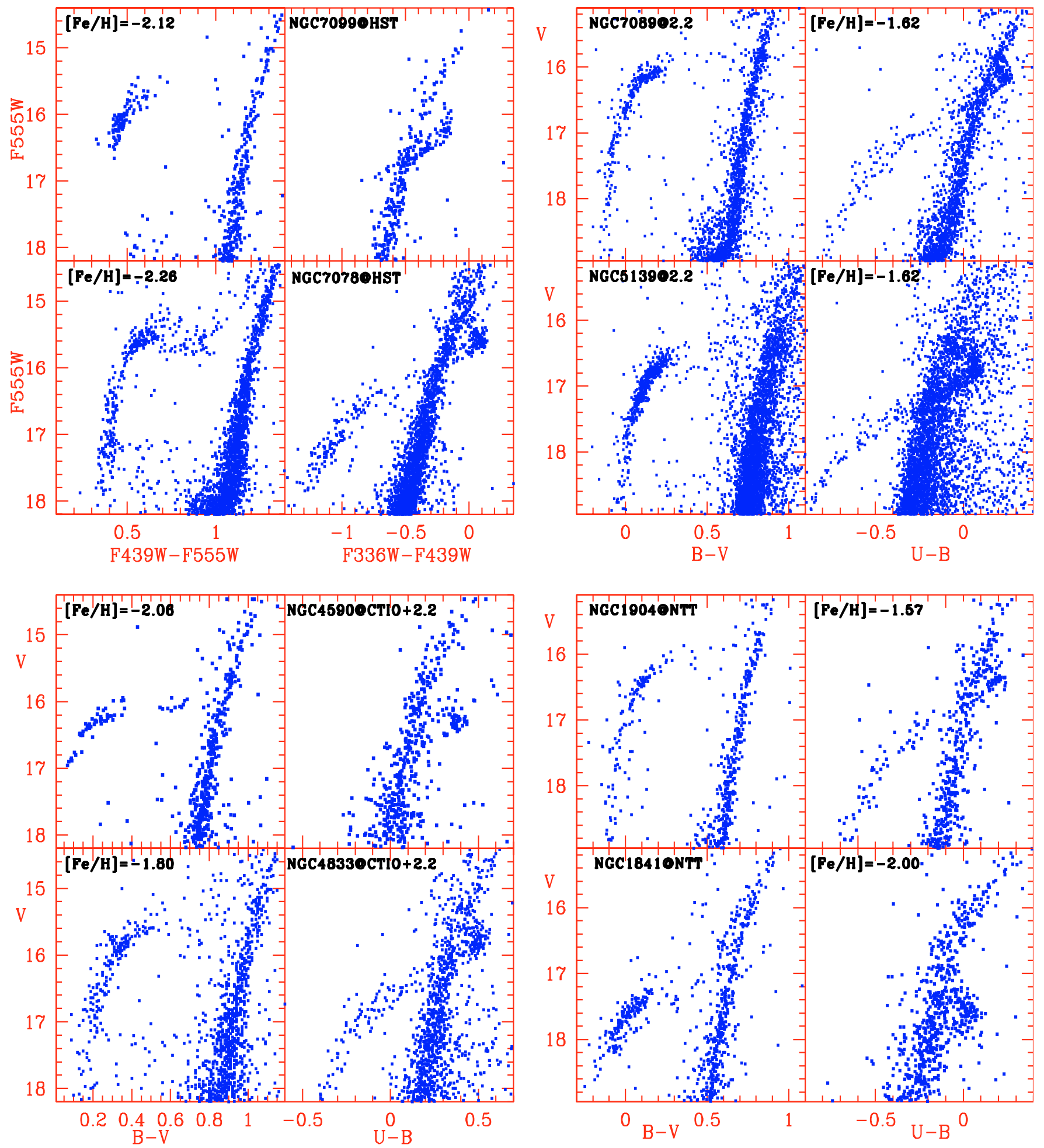

Fig. 1. $V,(B-V)$ and $V,(U-B)$ diagrams of a BHB cluster sample collected at 4 different telescopes. The diagrams in each panel were shifted for demonstration purposes. The reported metallicities are from the Harris on-line-catalog: http://physun.physics.mcmaster.ca/ harris/mwgc.dat (1996) as updated on February 2003, except for NGC 1841 (Alcaino et al. 1996).

incursion. Clearly, it is the combination of the non-standard $U$ and $B$ passband that creates the observed anomaly.

In Fig. 3 we show the normalized transmission curve of the $U B$ filters used at the $2.2 \mathrm{~m}$, the HST F336W and F439W filters and the Strömgren $u$ and $v$ filters. For two different metallicities $([\mathrm{Fe} / \mathrm{H}]=-1.8$ and -0.7$)$, we also show synthetic spectra (Castelli \& Kurucz 2003) for BHB stars, and for RGB stars with surface gravity and effective temperature appropriate for red giants at the level of the HB luminosity. When comparing a BHB spectrum with the transmission curves of different 


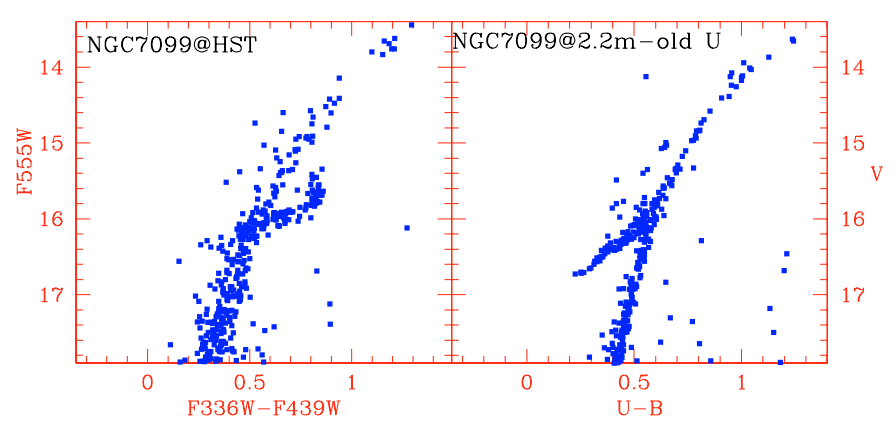

Fig. 2. Color-magnitude diagrams of NGC 7099 showing completely different HB morphologies as a function of the employed filters.

$U$ and $B$ filters, it is clear that the occurrence of the BHB red incursion is related to the Balmer discontinuity: $U$ filters peaking around $\lambda \simeq 3400 \AA$ do not encompass the Balmer jump. These filters collect less $U$ flux with respect to $U$ filters peaking at $\lambda \simeq 3650 \AA$. Consequently, the $U$ magnitudes of BHB stars are fainter and therefore their $(U-B)$ colors will be redder. On the other hand, RGB stars have a shallower Balmer jump, hence these are less affected by differences in $U$ filters. Also important is the broadness of the new- $B$ filter at the $2.2 \mathrm{~m}$ with respect to the old- $B$ filter. The blue side of the new- $B$ encompasses the Balmer jump, and is the cause of the anomalous morphology of the HB when the corresponding $B$ magnitudes are combined with the new- $U$ filter at the $2.2 \mathrm{~m}$.

A deeper understanding of the BHB red incursion can be achieved when considering the dependence of the $(U-B)$ color on stellar parameters such as surface gravity, effective temperature and chemical composition. The $U B V$ synthetic photometry from the ATLAS9 models (Castelli 1999, Fig. 11) shows that, for a given gravity, the $(U-B)$ index does not monotonically increase with decreasing temperature (see also Fig. 4). Instead, between $\sim 6750-5500 \mathrm{~K}$, the $(U-B)$ index shows a minimum which increases with decreasing metallicity, and is almost independent of gravity. Moving towards higher temperatures $(\geq 7000 \mathrm{~K})$, the $(U-B)$ index increases from the quoted minimum and reaches a maximum value, whose intensity depends on gravity but not on metallicity. The lower the gravity, the higher is the maximum. Moreover, the $(U-B)$ maximum moves from $\sim 7500 \mathrm{~K}$ for $\log g=2.0$ to about $9000 \mathrm{~K}$ for $\log g=5.0$. This means that in a metal-poor cluster, the $(U-B)$ index of a star with $T_{\text {eff }} \approx 8000 \mathrm{~K}$ and $\log g=3.0$ (i.e. an HB star) may be close to the maximum value and therefore exceeds the $(U-B)$ index of a RGB star with $T_{\text {eff }} \approx 5500-6000 \mathrm{~K}$.

This anomalous $(U-B)$ behavior is not encountered when the "standard" $U$-Buser filter or the old- $U$ filter at the $2.2 \mathrm{~m}$ telescope are used, simply because the difference between the maximum and the minimum in the $T_{\text {eff }}-(U-B)$ curve is not large enough to produce the red incursion. On the other hand, the difference between the minimum and maximum $(U-B)$ index becomes larger for $U$ filters with transmission curves shifted towards shorter wavelengths, to the point of producing the red incursion for the F336W filter on HST, Strömgren $u$ filter, and the new $U$ filter at the $2.2 \mathrm{~m}$ telescope.

To better explain the red incursion observed for low gravity stars, we replaced in the synthetic photometry (Castelli 1999) the $U$ filter from Buser (1978) and the $B$ filter from Ažusienis \& Straižys (1969) with the new $U$ and $B$ filters of the $2.2 \mathrm{~m}$ photometric system. Figure 4 shows that, for typical gravities of HB stars $(\log g=3.0)$, there is a large difference in the $T_{\text {eff }}-(U-B)$ curves for the two sets of filters; particularly at the $(U-B)$ maximum, where the difference is $\sim 0.2 \mathrm{mag}$. Figure 4 also shows that while the difference between the $(U-B)$ index of an HB star $(\log g=3.0)$ and an RGB star $(\log g=2.0)$ for the classical filters is approximately the same for all the temperatures, for the new $U B$ filters at the $2.2 \mathrm{~m}$ the $(U-B)$ color of an HB star is redder than the $(U-B)$ of an RGB star.

The effect we have just discussed is not new. It has been known as the "ultraviolet deficiency" of cool, BHB stars in the $(U-B, B-V)$ two-color diagram (see Markov et al. 2001 and references therein). Analyzing data for $6 \mathrm{GCs}$, Markov et al. compared the position of BHB stars with respect to i) a Population I sequence; and ii) a zero-age horizontal branch (ZAHB) two-color line, and quantified the $\Delta(U-B)$ deficiency. In analogy with our results, they also found that this observable is not common to all BHB clusters. Markov et al. (2001) argued that there exists a connection between this observable and cluster parameters. In particular, they showed how a satisfactory overlapping of the observed and theoretical sequences is achieved when using low-gravity ( $\log g \leq 2.0)$ atmospheric models.

As expected from the above discussion, using a $U$ filter-set showing the BHB red incursion (e.g. the F336W filter on HST or the "new" $U$ filter at the $2.2 \mathrm{~m}$ telescope) not all BHB clusters show the red incursion. It is clearly present in a metalpoor cluster like NGC 7078, whereas it is completely missing in an intermediate metallicity cluster like NGC 6205 (see also Fig. 6). Due to the small sample of clusters, we can neither observationally quantify the dependence of the effect on the cluster metal content nor precisely estimate the cutoff metallicity (if any) beyond which no incursion is observed. The fact that the HB of NGC $1904\left([\mathrm{Fe} / \mathrm{H}] \sim-1.6, E_{B-V}=0.01\right)$ shows a red incursion while NGC $6205([\mathrm{Fe} / \mathrm{H}] \sim-1.5$, $\left.E_{B-V}=0.02\right)$ does not show any incursion could suggest that this effect might be present mainly in clusters with metallicities lower than $[\mathrm{Fe} / \mathrm{H}] \approx-1.5$. Inspection of the HST F555W, $(\mathrm{F} 336 \mathrm{~W}-\mathrm{F} 439 \mathrm{~W})$ diagram of NGC $6388([\mathrm{Fe} / \mathrm{H}] \simeq-0.60$, showing no indication of the incursion) suggests that the effect is not present in the CMDs from broad band photometry of high metallicity BHB clusters. In view of the strong dependence of the effect on the filter transmission curve, only a photometrically homogeneous observational data set covering a large metallicity range will allow us to characterize the phenomenon and investigate whether this feature can be used to constrain other cluster parameters, like metallicity, distance, and reddening.

The suggestion for a metallicity threshold of the incursion relies on a set of images collected with filters that are supposed to reproduce the standard $U B V$ Johnson bands at 3 widely used telescopes: HST, NTT and the $2.2 \mathrm{~m}$. It must be noted that the red incursion effect is clearly visible also in other bands, like the Strömgren intermediate ones. However, the metallicity threshold is not present in all the photometric systems. Indeed, theoretical isochrones and ZAHBs (from the 


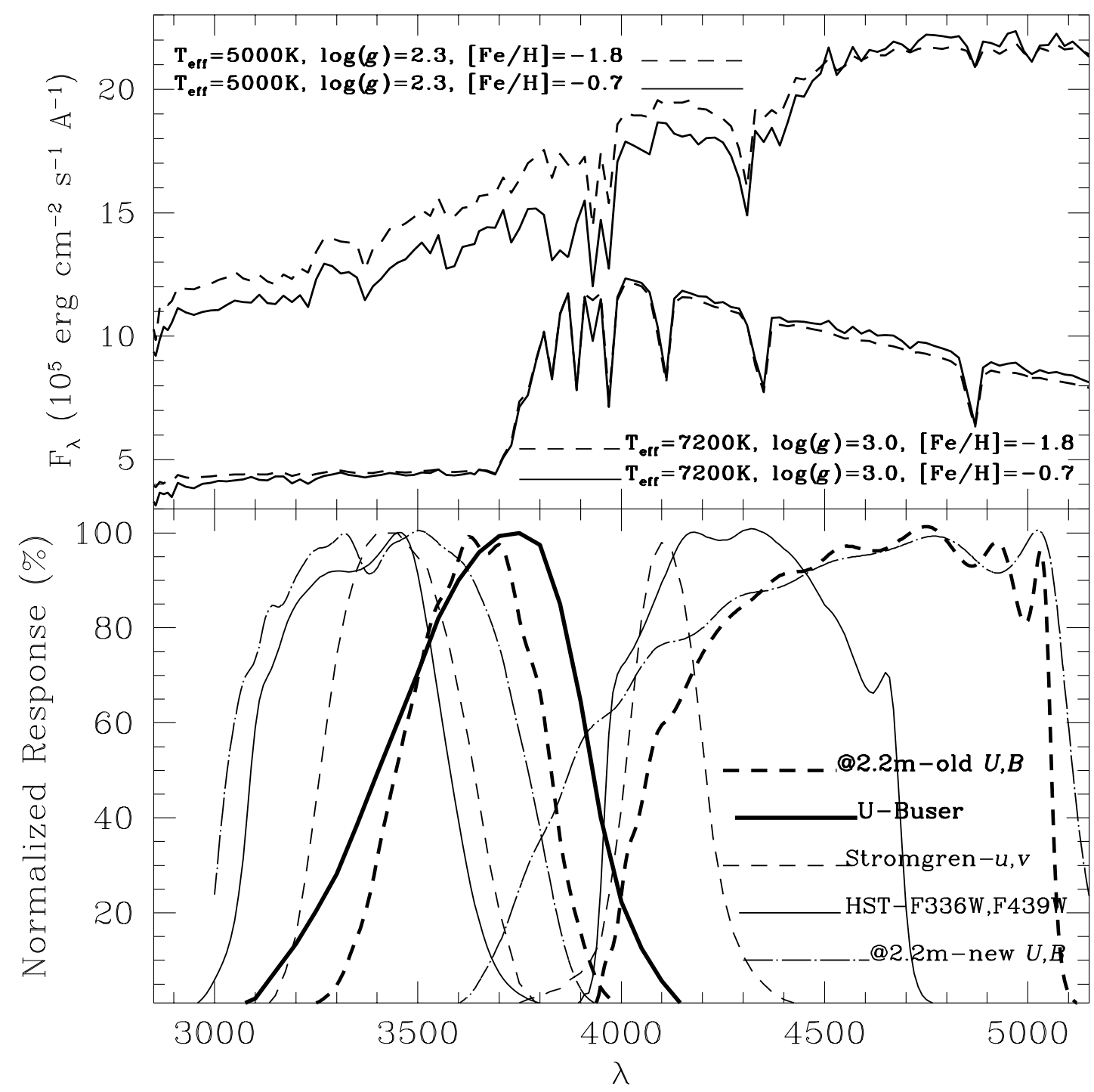

Fig. 3. Upper panel shows a comparison between different stellar spectra for two different choices of the star metallicity, gravity and effective temperatures, representative of BHB stars (lower spectra) and RGB structures (upper spectra) with the same bolometric luminosity of HB objects. The "RGB" spectra has been shifted upwards for demonstration purposes. Lower panel shows the normalized transmission curves of different $U B$ filters.

Cassisi \& Salaris 1997 library) show the incursion in the Strömgren $M_{V},(u-v)$ plane even at metallicities of $Z=0.006$, representative of metal-rich GCs.

Figure 5 helps us to understand the metallicity effect of the BHB red incursion. For the HST flight and the Strömgren photometric systems we have plotted the following three color differences as a function of metallicity: (1) $\Delta(\mathrm{HB}-\mathrm{RGB})$ measures the extent of the incursion, i.e. the difference in color between the reddest $\mathrm{HB}$ point and the RGB color at the level of the reddest HB point; (2) $\Delta\left(\mathrm{HB}-\mathrm{HB}_{Z}=0.0001\right)$ is the difference in color between the reddest $\mathrm{HB}$ point for the quoted metallicities and that for $Z=0.0001$; and lastly, (3) $\Delta\left(\mathrm{RGB}-\mathrm{RGB}_{Z=0.0001}\right)$ is the difference between the RGB colors (at the level of the reddest HB point for the three indicated metallicities) and that for $Z=0.0001$. There are two important conclusions that can be drawn from Fig. 5. First of all, we confirm that, while the red incursion of the HB is present at least up to metallicities of $Z=0.006$ in the Strömgren photometric system, the phenomenon is expected to disappear for clusters more metal rich than $Z=0.001$ in the HST flight system, where the corresponding $\Delta(\mathrm{RGB}-\mathrm{HB})$ becomes negative. The second important evidence disclosed by Fig. 5 is that the extension of the red incursion (and its disappearance for more metalrich clusters in the broad band CMDs) is due to the fact that the RGB becomes redder and redder at increasing metallicity, while the color of the reddest HB point remains more less the same at varying the cluster metallicity.

In Fig. 6, we also compare the observed WFPC2/ HST F555W, (F336W-F439W) CMDs of NGC 7078 and NGC 6205 with the isochrones from Cassisi \& Salaris (1997) transformed into the HST flight system. The data have not been calibrated, but the calibration to the HST flight system is simply a zero point translation, and does not affect the overall morphology of the CMD. It is worth noticing that the models reproduce very well the BHB "elbow" morphology, and, most importantly, reproduce the BHB red incursion when present. 


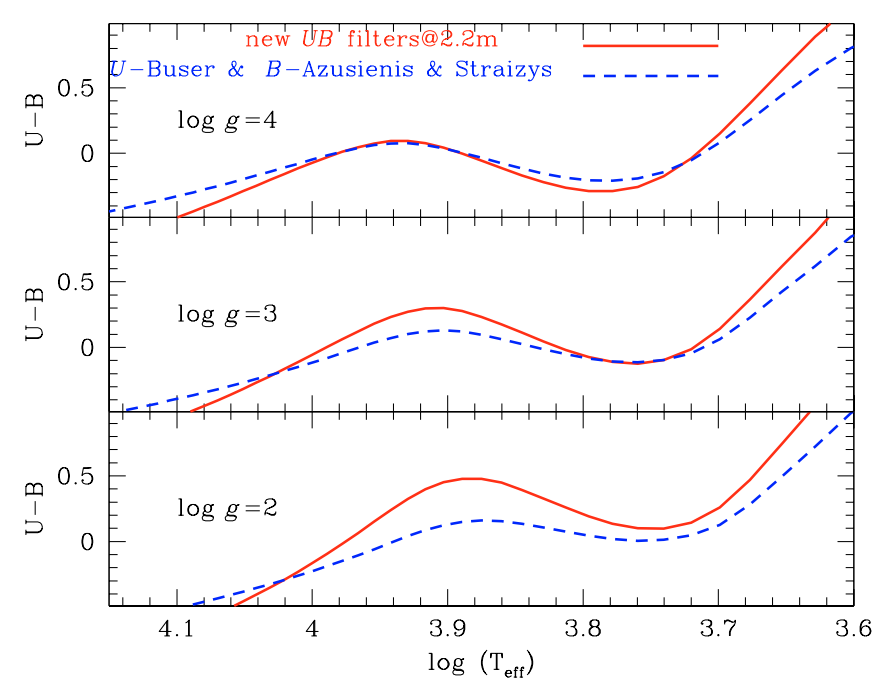

Fig. 4. Computed $T_{\text {eff }}-(U-B)$ relations for $[\mathrm{M} / \mathrm{H}]=-2.0$, different $\log g$, and different $U B$ filters. Dashed lines are the relations obtained using the $U$-Buser (1978) and B-Ažusienis \& Straižys (1969) filters, continuous lines are those obtained using the new $U B$ filters at the $2.2 \mathrm{~m}$ telescope.

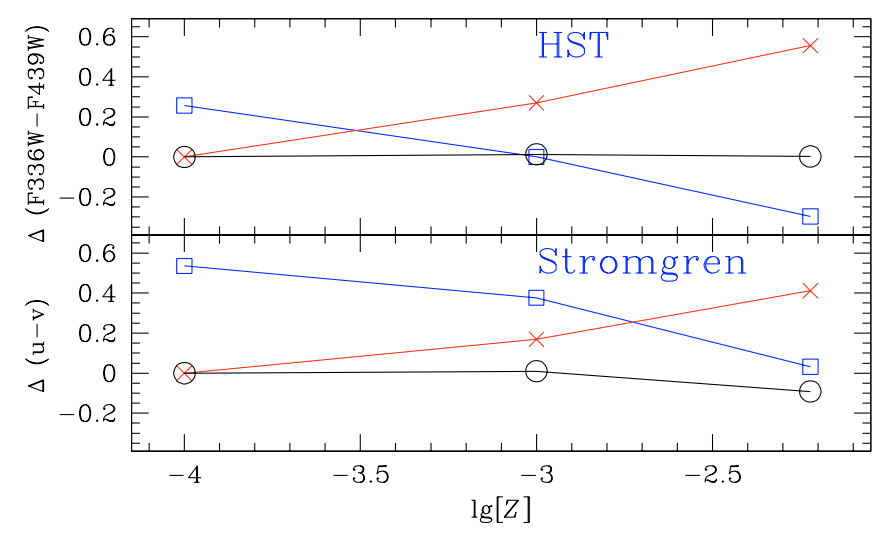

Fig. 5. RGB and $\mathrm{HB}$ color differences, at the level of the reddest HB incursion, estimated for three isochrones $(Z=0.0001,0.001$, and 0.006) in the HST flight and the Strömgren photometric systems. Open squares measure the extent of the incursion ( $\Delta$ color between the $\mathrm{HB}$ and the RGB), crosses mark the RGB color differences of the three isochrones with respect to the $Z=0.0001$ one, and open circles mark the $\mathrm{HB}$ color differences with respect to the $Z=0.0001$ isochrone.

We have also compared the models with the CMDs from ground-based photometry for those clusters showing the BHB red incursion. In this case the models are not able to reproduce such a feature at all. This is a consequence of the fact that the $U$-filter used to derive the color- $T_{\text {eff }}$ relations (adopted for transforming the models from the theoretical to the empirical plane) is the $U$-Buser filter, clearly inadequate to fit data obtained with "non-standard" $U$ filters (cf. Fig. 3).

This occurrence strongly reinforces the need for appropriate color- $T_{\text {eff }}$ relations, based on exactly the same filter passband used in the observations, in order to realize a meaningful comparison between theory and observations. Most importantly, the anomalous CMD morphology (produced by $U$ filters whose transmission is far from the standard ones) calls attention to another problem, often ignored by those in

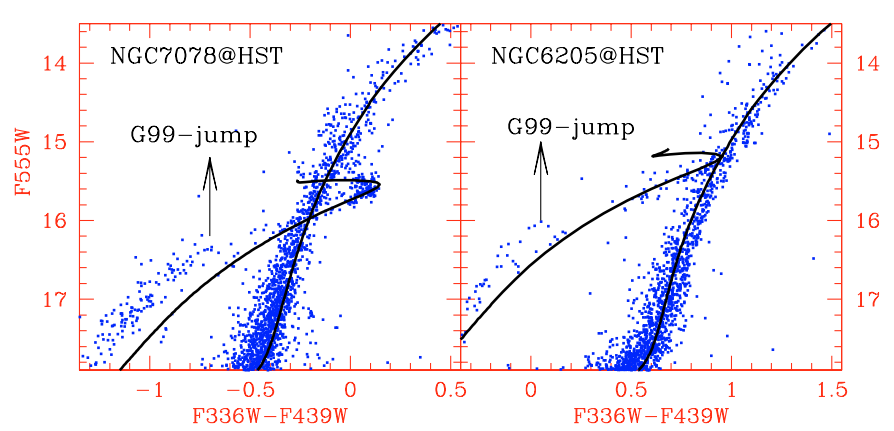

Fig. 6. HST F555W, (F336W-F439W) diagrams of NGC 7078 and NGC 6205, along with theoretical isochrones and ZAHBs (Cassisi \& Salaris 1997) with $Z=0.0001$ (appropriate for NGC 7078) and $Z=$ 0.001 (appropriate for NGC 6205).

charge of the development and maintenance of imaging instruments: filter passbands must be as close as possible to the bands they claim to reproduce. This simple rule makes the calibration to the standard photometric system more accurate, and avoids the production of apparently un-physical features in the observed CMDs.

\section{Summary and final remarks}

In the present work, we show the existence of a curious feature in the $V,(U-B)$ CMDs of some GCs. Quite surprisingly, in some observed CMDs, hot HB stars $\left(6000 \lessgtr T_{\text {eff }}(\mathrm{K}) \lesssim 10000\right)$ become redder than the much cooler RGB. An investigation of this effect has shown the following:

- The size and the presence itself of this feature is strongly dependent on the passband of the adopted $U$ filter. In particular, $U$ filters that do not encompass the Balmer jump show the BHB red incursion.

- The $B$-filter passband also acts to cause the anomaly.

- This feature is due to the peculiar dependence of the $U$ and $B$ filters on star effective temperature, gravity, and metallicity.

- Clusters more metal-rich than $[\mathrm{Fe} / \mathrm{H}] \simeq-1.5$ do not show any BHB red incursion in the broad band Johnson and HST flight system, while there is no metallicity threshold in Strömgren $u v b y$ photometry for metallicities lower than 0.006 .

- Evolutionary models using the correct filter passband, when transformed to the observational plane, finely reproduce the observed HB morphology.

We report this anomalous HB morphology to point out the importance of having observational photometric systems that accurately reproduce the standard ones. Unfortunately, the fact that the anomalous CMDs shown in this paper have been obtained from data acquired at some of the most widely used instruments for astronomical imaging shows how this problem is yet to be addressed.

Acknowledgements. We warmly thank the referee, F. Grundahl, for the useful comments which helped to improve the paper. 
In particular, we thank him for pointing out the presence of the effect described in this paper also in the CMDs from Strömgren photometry. We also thank G. Bono and V. Castellani for useful discussions on this matter. Y.M thanks S. Lucatello for useful discussions on stellar spectra. L.R.B., Y.M., S.O., G.P., and A.R.B. acknowledge the support of the MIUR (Cofin2001). S.C acknowledges financial support by MIUR (Cofin2002).

\section{References}

Alcaino, G., Liller, W., Alvarado, F., et al. 1996, AJ, 112, 2020

Ažusienis, A., \& Straižys, V. 1969, Soviet Astron., 13, 316

Brown, T. M., Sweigart, A. V., Lanz, T., Landsman, W. B., \& Hubeny, I. 2001, ApJ, 562, 368

Buser, R. 1978, A\&A, 62, 411

Cassisi, S., \& Salaris, M. 1997, MNRAS, 285, 593

Castelli, F. 1999, A\&A, 346, 564

Castelli, F., \& Kurucz 2003, Modelling of Stellar Atmospheres, IAU Symp. 210, ed. N. E. Piskunov, W. W. Weiss, \& D. F. Gray, PASP Conf. Ser., in press
D’Cruz, N. L., Noella, L., D'Connell, R. W., et al. 2000, ApJ, 530, 352

Germany, L. 2002, The Messenger, 107, 13

Grundahl, F., Catelan, M., Landsman, W. B., Stetson, P. B., \& Andersen, M. I. 1999, ApJ, 524, 242 [G99]

Harris, W. E. 1996, AJ, 112, 1487

Johnson, H. L. 1955, Ann. Astrophys., 18, 292

Kravtsov, V., Ipatov, A., Samus, N., et al. 1997, A\&AS, 125, 1

Landolt, A. U. 1992, AJ, 104, 372

Markov, H. S., Spassova, N. M., \& Baev, P. V. 2001, MNRAS, 326, 102

Melbourne, J., Sarajedini, A., Layden, A., \& Martins, D. H. 2000, AJ, 120, 3127

Momany, Y., Piotto, G., Recio-Blanco, A., et al. 2002, ApJ, 576, L65 Piotto, G., King, I. R., Djorgovski, S. G., et al. 2002, A\&A, 391, 945 Stetson, P. B. 1994, PASP, 106, 250

Valdes, F. G. 1998, Astronomical Data Analysis Software and Systems VII, ASP Conf. Ser., 145, 53

Walker, A. R. 1994, AJ, 108, 555 